\title{
Neuronal Glutamate Uptake Contributes to GABA Synthesis and Inhibitory Synaptic Strength
}

\author{
Gregory C. Mathews ${ }^{1,2}$ and Jeffrey S. Diamond ${ }^{1}$ \\ ${ }^{1}$ Synaptic Physiology Unit, National Institute of Neurological Disorders and Stroke, National Institutes of Health, Bethesda, Maryland 20892-4066, and \\ ${ }^{2}$ Department of Neurology, Johns Hopkins University School of Medicine, Baltimore, Maryland 21287
}

\begin{abstract}
Neurons must maintain a supply of neurotransmitter in their presynaptic terminals to fill synaptic vesicles. GABA is taken up into inhibitory terminals by transporters or is synthesized from glutamate by glutamic acid decarboxylase. Here we report that glutamate transporters supply GABAergic terminals in the hippocampus with glutamate, which is then used to synthesize GABA for filling synaptic vesicles. Glutamate transporter antagonists reduced IPSC and miniature IPSC (mIPSC) amplitudes, consistent with a reduction in the amount of GABA packaged into each synaptic vesicle. This reduction occurred rapidly and independently of synaptic activity, suggesting that modulation of vesicular GABA content does not require vesicle release and refilling. Raising extracellular glutamate levels increased mIPSC amplitudes by enhancing glutamate uptake and, consequently, GABA synthesis. These results indicate that neuronal glutamate transporters strengthen inhibitory synapses in response to extracellular glutamate. This modulation appears to occur under normal conditions and may constitute a negative feedback mechanism to combat hyperexcitability.
\end{abstract}

Key words: glutamate transporters; synaptic vesicles; GABA; metabolism; inhibition; hippocampus

\section{Introduction}

Glutamate is an essential component of synaptic transmission in the CNS, both at excitatory synapses, where it is the neurotransmitter, and at inhibitory synapses, where it serves as the substrate for GABA synthesis. In excitatory neurons, glutamate is synthesized either from glutamine or from glucose metabolites. Both pathways rely on astrocytes, which supply neurons with essential substrates (for review, see Hertz et al., 1999). Glutamate is also taken up into excitatory presynaptic terminals by high-affinity transporters (for review, see Danbolt, 2001). In inhibitory neurons, all GABA, except that taken up through transporters, is synthesized by decarboxylation of glutamate by glutamic acid decarboxylase (GAD; for review, see Martin and Tobin, 2000). It is not known whether glutamate metabolism in inhibitory terminals mirrors that in excitatory terminals, although it has been proposed that glutamate may be taken up into inhibitory terminals by presynaptic transporters (Rothstein et al., 1994; Sepkuty et al., 2002).

High-affinity glutamate transporters function primarily to maintain a low concentration of glutamate in the extracellular space. Although some subtypes [glutamate transporter 1 (GLT-1) and glutamate-aspartate transporter] are localized predominantly to astrocytes, others [excitatory amino acid carrier 1 (EAAC1) and excitatory amino acid transporter 4] are expressed mostly by neurons (Rothstein et al., 1994). Both glial and neuronal transporters may be localized to play specific homeostatic roles, although a lack of selective inhibitors (except for GLT-1) has hindered investigation of unique physiological roles for these

Received 0ct. 11, 2002; revised Dec. 4, 2002; accepted Dec. 20, 2002.

This work was supported by the National Institute of Neurological Disorders and Stroke Intramural Research Program. We thank the members of our laboratory for valuable discussions.

Correspondence should be addressed to Dr. Jeffrey S. Diamond, Synaptic Physiology Unit, National Institute of Neurological Disorders and Stroke, National Institutes of Health, 36 Convent Drive, Room 2C09, Bethesda, MD 20892-4066. E-mail: diamondj@ninds.nih.gov.

Copyright $\odot 2003$ Society for Neuroscience $\quad 0270-6474 / 03 / 232040-09 \$ 15.00 / 0$ transporters. Perisynaptic transporters may be positioned to prevent excitotoxicity (Rothstein et al., 1996), to limit diffusion of glutamate between excitatory synapses (Asztely et al., 1997; Lozovaya et al., 1999; Carter and Regehr, 2000; Diamond, 2001; Arnth-Jensen et al., 2002), or to supply the terminal with neurotransmitter (Gundersen et al., 1993). Transporters used by excitatory terminals have not been identified but may be a GLT-1-like subtype (Danbolt, 2001; Chen et al., 2002). Anatomical data indicate that the neuronal subtype EAAC1 colocalizes with GAD (Conti et al., 1998) and is located at inhibitory terminals (Rothstein et al., 1994; He et al., 2000). Reducing expression of EAAC1 is associated with a decrease in both tissue GABA levels and de novo synthesis from glutamate, which may lead to neuronal hyperexcitability and epilepsy (Sepkuty et al., 2002).

We show here that presynaptic glutamate transporters are involved in the bidirectional regulation of inhibitory synaptic transmission. Inhibiting neuronal glutamate transporters reduced $\mathrm{GABA}_{\mathrm{A}}$ receptor $\left(\mathrm{GABA}_{\mathrm{A}} \mathrm{R}\right)$-mediated IPSCs and miniature IPSCs (mIPSCs), suggesting that GABA synthesis and, consequently, vesicular filling were reduced. These effects did not depend on astrocytic glutamate metabolism. They also occurred independently of synaptic activity, suggesting that transmitter concentration can be modulated in vesicles that are ready to be released. In addition, enhancing glutamate uptake increased the amplitude of miniature IPSCs. The bidirectional regulation of GABAergic transmission was prevented by an inhibitor of GAD, verifying that the uptake-dependent effects required GABA synthesis. Our findings that glutamate transporters on inhibitory terminals contribute to GABA release suggest that a compensatory or protective mechanism allows extracellular glutamate to strengthen inhibitory synapses.

\section{Materials and Methods}

Electrophysiology. Whole-cell patch-clamp recordings from CA1 pyramidal neurons were made in transverse hippocampal slices ( $400 \mu \mathrm{m}$ thick) 
made from postnatal day $10-14 \mathrm{~d}$ rats as described previously (Sakmann and Stuart, 1995). Slices were stored in and perfused with a solution containing (in $\mathrm{mm}$ ): $119 \mathrm{NaCl}, 2.5 \mathrm{KCl}, 2.5 \mathrm{CaCl}_{2}, 1.3 \mathrm{MgCl}_{2}, 1$ $\mathrm{NaH}_{2} \mathrm{PO}_{4}, 26.2 \mathrm{NaHCO}_{3}$, and 11 glucose, bubbled with $95 \% \mathrm{O}_{2}$ and $5 \%$ $\mathrm{CO}_{2}$ at room temperature. All solutions also contained $10 \mu \mathrm{M} 6,7-$ dinitroquinoxaline-2,3-dione (DNQX) and $5 \mu \mathrm{M}$ MK-801 (dizocilpine maleate) to block glutamatergic responses ( $20 \mu \mathrm{M}$ DNQX was used when exogenous glutamate was applied). Patch pipettes (resistance, 2-4 M $\Omega$ ) were filled with (in mM): $130 \mathrm{CsCl}, 10$ EGTA, 10 HEPES, 2 Mg-ATP, 0.2 Na-GTP, and 1 QX-314 (lidocaine $N$-ethyl bromide), titrated to $\mathrm{pH} 7.4$ with $\mathrm{CsOH}$. Using an upright fixed stage microscope (Zeiss, Thornwood, $\mathrm{NY}$ ), pyramidal neurons were identified under video observation using infrared differential interference contrast imaging. Unless indicated otherwise (see Figs. $4 C, 5 D)$, voltage-clamp recordings $\left(V_{\text {hold }}=-60 \mathrm{mV}\right.$, uncorrected for junction potential) were made at room temperature using an Axopatch 1D amplifier (Axon Instruments); currents were filtered at $2 \mathrm{kHz}$, sampled at $5 \mathrm{kHz}$, and digitally stored for off-line analysis (IGOR Pro software). Access resistance was monitored throughout the experiment, and data were discarded when $>10 \%$ change occurred. IPSCs were evoked at $8-15 \mathrm{sec}$ intervals with a bipolar stainless steel electrode placed in the stratum oriens (constant current stimulation, $25-150 \mu \mathrm{A} ; 100 \mu \mathrm{sec}$ duration). When required, D,L-threo- $\beta$-hydroxyaspartic acid (THA, $300 \mu \mathrm{M}$ ) and dihydrokainic acid (DHK, $300 \mu \mathrm{M}$; Ocean Produce International) were dissolved in the recording solutions just before the experiment; D,L-threo- $\beta$-benzyloxyaspartic acid (TBOA, $30 \mu \mathrm{M}$ ) was diluted from a stock solution. Sucrose $(500 \mathrm{~mm})$ and GABA $(10 \mu \mathrm{M})$ were added to the control solution and were pressure-applied (2-3 sec puffs, $4-5$ psi) through a patch pipette. When effects of THA on puffed GABA responses were tested, THA was included in the perfusion and pipette solutions. In hyperosmotic stimulation experiments, 1 min was allowed between applications to ensure refilling of the vesicular pool, and responses were quantitated by integrating the current over the duration of the sucrose application. For studies involving inhibition of GAD or glutamine synthetase, mercaptopropionic acid (MPA, $250 \mu \mathrm{M}$ ) or methionine sulfoximine (MSO, $1.5 \mathrm{~mm}$ ) was included in solutions used for storage (3-7 hr before recording) and perfusion of slices.

IIPSC recording and analysis. Electrically evoked mIPSCs were obtained as described above, except that calcium in the extracellular solution was replaced with strontium, giving rise to numerous asynchronous events after each stimulus (Goda and Stevens, 1994). Stimuli were delivered every $8-10 \mathrm{sec}$, and events were collected in the $718 \mathrm{msec}$ after each stimulus, excluding the initial $100 \mathrm{msec}$. Raw traces were differentiated and smoothed; events exceeding a slope threshold (chosen to detect even the smallest and slowest events) were then visually examined. Only single events occurring from a level baseline were selected for analysis. Responses to $60-70$ stimuli, resulting in $\sim 200$ events, were elicited in each condition in each cell. When drug effects were measured, $5 \mathrm{~min}$ of wash-in was allowed before mIPSCs were collected. To compare between cells, individual event amplitudes in each cell were normalized to the mean amplitude in a control. Decay time constants were estimated by fitting a single exponential to the decay phase of the averaged mIPSC in each cell.

Unless indicated otherwise, data are expressed as mean $\pm \mathrm{SD}$, and $p$ values were derived from paired $t$ tests, with significance concluded at $p<0.05$. For pooled mIPSC amplitudes, which were not normally distributed, the Mann-Whitney $U$ test was used. In graphs illustrating responses over time from a collection of experiments (see Figs. $1 C, 3 D$ ) and in cumulative probability histograms (see Figs. 4-6), error bars indicate SEM.

\section{Results}

\section{Glutamate transporter blockade reduces electrically evoked IPSCs}

Inhibitory interneurons are abundant in stratum oriens (SO) of area CA1, where distinct subtypes make either axosomatic or axodendritic synapses onto excitatory pyramidal neurons (McBain and Fisahn, 2001). In juvenile hippocampal slices (postnatal days 10-14), electrical stimulation in SO elicited monosynaptic $\mathrm{GABA}_{\mathrm{A}} \mathrm{R}$-mediated IPSCs, which were recorded under whole-cell voltage-clamp conditions $\left(V_{\text {hold }}=-60 \mathrm{mV}\right)$ in the presence of glutamate receptor antagonists (see Materials and Methods). Inward IPSCs (attributable to symmetrical chloride concentrations) could be blocked completely by SR95531 (gabazine) $(10 \mu \mathrm{M})$, a selective $\mathrm{GABA}_{\mathrm{A}} \mathrm{R}$ antagonist (Fig. $\left.1 \mathrm{~A}\right)$. To examine the effects of glutamate uptake on IPSCs, we applied THA $(300 \mu \mathrm{M})$, a competitive inhibitor of both neuronal and astrocytic glutamate transporters (Arriza et al., 1994). THA $(300 \mu \mathrm{M})$ does not completely block glutamate uptake in hippocampal slices (Bergles and Jahr, 1997), because it is also transported, thereby reducing its effective concentration. THA reduced evoked IPSC amplitudes to $74 \pm 22 \%$ of control $(p<0.001 ; n=16$ cells; Fig. $1 B, C, H)$; this effect recovered only partially on washout. A nontransported inhibitor, TBOA (30 $\mu \mathrm{M}$; Shimamoto et al., 1998), caused a similar reduction in (and incomplete recovery of) IPSC amplitude (to $71 \pm 10 \%$ of control; $p=0.005 ; n=4$ cells; Fig. $1 D, H)$, suggesting that THA did not exert its effect intracellularly (e.g., by inhibiting vesicular transporters). To test whether the effects of glutamate transporter inhibition on IPSCs were mediated by the GABA synthetic pathway, THA was applied to slices that had been preincubated for 3-7 hr with the GAD inhibitor MPA (250 $\mu \mathrm{M}$; Lamar, 1970). IPSC amplitudes were gradually reduced after application of MPA without reaching a plateau in 30 min (data not shown); after several hours of preincubation with MPA, stronger stimuli were required to evoke IPSCs that were smaller on average than those of controls $(-380 \pm 177 \mathrm{pA}$ in MPA vs $-711 \pm 447 \mathrm{pA}$ ). In MPA-treated slices, THA had no significant effect on IPSC amplitudes $(110 \pm 20 \%$ of control; $p=$ $0.17 ; n=5$; Fig. $1 E, H)$, suggesting that transported glutamate acts as a substrate for GABA synthesis.

Glutamate taken up by astrocytes may be converted to glutamine and returned to neurons, a cycle requiring the glialspecific enzyme glutamine synthetase (Martinez-Hernandez et al., 1977). It is possible that THA, a blocker of astrocytic and neuronal glutamate transporters, reduces IPSC amplitudes by disrupting this intermediary metabolism. We tested this possibility with MSO, an inhibitor of glutamine synthetase (Sellinger, 1967). MSO (1.5 mM) prevents conversion of glutamate to glutamine, thereby trapping glutamate in glial cells and decreasing glutamate levels in synaptic terminals twofold (Laake et al., 1995). In slices that had been preincubated for 3-7 hr with $1.5 \mathrm{~mm}$ MSO, field EPSPs were reduced by $35 \%$ compared with responses elicited under identical conditions in control slices (control, $0.51 \pm 0.21 \mathrm{mV} ; n=12 ; \mathrm{MSO}, 0.33 \pm 0.16 \mathrm{mV} ; n=12 ; p=0.02$, unpaired $t$ test), indicating that glutamate metabolism was compromised. In MSO-treated slices, however, THA still reduced IPSC amplitudes to $71 \pm 17 \%$ of control ( $p=0.03 ; n=7$ cells; Fig. $1 F, H$ ), indicating that glutamate transporter antagonists do not reduce IPSCs through actions on astrocytic glutamate uptake.

Interneurons in stratum radiatum (SR) express higher levels of the GABA transporter GAT-1 than interneurons in SO (Engel et al., 1998) and therefore may rely less than SO interneurons on GABA synthesis to fill synaptic vesicles. Nonetheless, THA also reduced IPSCs elicited by SR stimulation $(55 \pm 27 \%$ of control; $p=0.014 ; n=5$ cells; Fig. $1 G, H)$, indicating that a role for presynaptic glutamate uptake is not unique to SO interneurons. Responses in all subsequent experiments were elicited by stimulation in SO.

These results suggest that glutamate uptake into inhibitory synaptic terminals is an important source of GABA for filling synaptic vesicles. If vesicles remain filled to a "set point" until being released, one would expect that the effects of transporter 

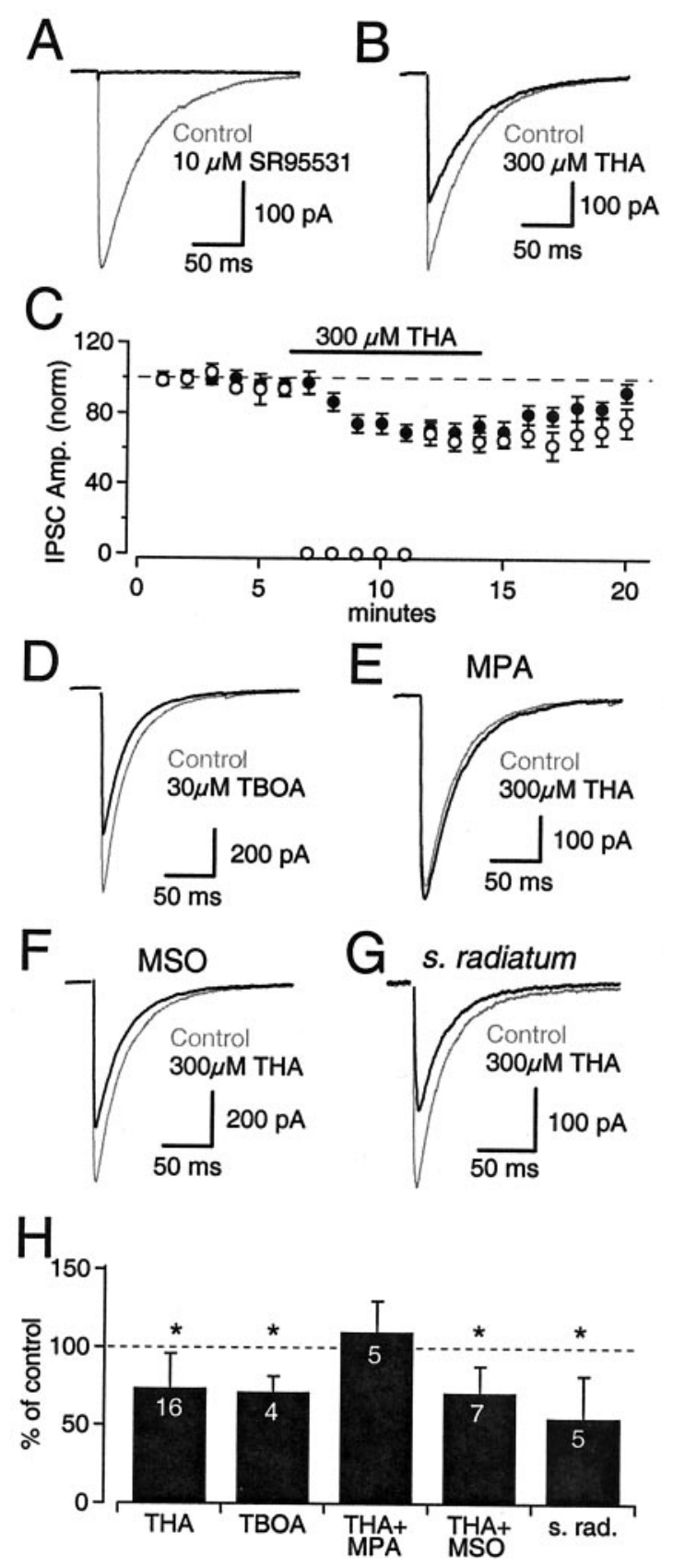

Figure 1. Inhibition of glutamate transporters reduces electrically evoked IPSCS. A, Evoked IPSC recorded from a CA1 pyramidal cell $\left(V_{\text {hold }}=-60 \mathrm{mV}\right.$ ) in control solution ( $\left.g r a y\right)$ and in the presence of the GABA ${ }_{A} R$ antagonist SR95531 (black). B, Evoked IPSCs in control solution (gray) and in the presence of $300 \mu \mathrm{m}$ THA (black), a nonselective glutamate transporter antagonist. $C$, Normalized IPSC amplitudes [Amp. (norm)] during experiments testing the effects of THA ( $n=$ 16 cells) with stimulation either continued (filled circles) or suspended (open circles) during wash-in. Error bars indicate SEM. D, IPSC in control solution ( gray) and in the presence of $30 \mu \mathrm{M}$ TBOA (black), a nonselective, nonsubstrate inhibitor of glutamate uptake. E, IPSC in control solution (gray) and in the presence of $300 \mu \mathrm{M}$ THA (black) after inhibition of GABA synthesis with $250 \mu \mathrm{M}$ MPA, a GAD inhibitor. F, IPSC in control solution (gray) and in the presence of 300 $\mu \mathrm{M}$ THA (black) after inhibition of astrocytic glutamine synthetase with $1.5 \mathrm{~mm}$ MSO. G, IPSCS recorded from a CA1 pyramidal cell after stimulation in stratum radiatum (s. radiatum) in control solution (gray) and in the presence of $300 \mu \mathrm{m}$ THA (black). H, Summary of effects of glutamate transporter antagonists on IPSC amplitude in various conditions. White numbers in bars indicate the numbers of cells tested. Error bars indicate SD. Asterisks denote a statistically significant difference compared with control $(p<0.05)$. s. rad., Stratum radiatum. blockade would not be evident until the pool of filled vesicles was released and recycled. Evidence suggests, however, that the vesicular transmitter concentration reflects a dynamic equilibrium between the vesicle and the synaptic terminal (Williams, 1997), allowing transmitter levels in vesicles to be modulated before release. To distinguish between these possibilities, in a separate set of experiments stimulation was suspended for the first $5 \mathrm{~min}$ of THA application to test whether the effect of THA required synaptic activity. When stimulation was resumed, a similar reduction in IPSC amplitude was observed ( $70 \pm 16 \%$ of control; $p=0.03 ; n=5$ cells; Fig. $1 C$ ) as when stimulation was not interrupted, suggesting that the effects of blocking glutamate transporters do not require release and subsequent recycling of synaptic vesicles.

\section{THA does not affect release probability or postsynaptic $\mathrm{GABA}_{\mathrm{A}} \mathrm{Rs}$}

Activation of presynaptic metabotropic glutamate receptors (mGluRs) inhibits GABA release at some synapses (Desai et al., 1994; Gereau and Conn, 1995; Mitchell and Silver, 2000); blocking glutamate transporters enhances mGluR activation at both excitatory and inhibitory synapses (Fitzsimonds and Dichter, 1996; Brasnjo and Otis, 2001; Reichelt and Knopfel, 2002). To test whether the decreased IPSC amplitude in the presence of THA involved mGluR activation, THA was applied in the presence of group I and II and group III mGluR antagonists [( $R S)$ - $\alpha$-methyl4-carboxyphenylglycine (MCPG), $500 \mu \mathrm{M}$; and (RS)- $\alpha$ cyclopropyl-4-phosphonophenylglycine (CPPG), $200 \mu \mathrm{M}$, respectively]. THA nonetheless reduced the IPSC amplitude (to $81 \pm 3 \%$ of control; $p=0.02 ; n=4$; Fig. $2 A$ ). The effect in the presence of mGluR antagonists was not significantly different from that in their absence ( $p=0.5$, unpaired $t$ test; Fig. $2 B)$, suggesting that mGluRs do not mediate the IPSC reduction. THA affected neither paired pulse depression (observed in $2.5 \mathrm{~mm}$ calcium: $105 \pm 13 \%$ of control; $n=6$; $p=0.37$ ) nor paired pulse facilitation (observed in $1 \mathrm{~mm}$ external calcium: $99 \pm 26 \%$ of control; $n=5 ; p=0.99$ ) of IPSCs (Fig. $2 C, D$ ), suggesting that the reduction of IPSC amplitude was not attributable to a change in release probability. Moreover, THA did not reduce the response to exogenously applied GABA (puff responses in THA were $102 \pm 9 \%$ of control; $p=0.9 ; n=3$; Fig. $2 E, F$ ), indicating that the drug did not affect postsynaptic $\mathrm{GABA}_{\mathrm{A}}$ receptors.

\section{THA reduces hyperosmotically evoked IPSCs}

The above results suggest that THA acts presynaptically to reduce IPSC amplitude without altering release probability. If glutamate uptake contributes to the process by which presynaptic vesicles are filled with GABA, one would predict that blocking transporters with THA would reduce vesicular filling and diminish the postsynaptic response to release of the entire pool of synaptic vesicles. Application of hyperosmolar extracellular solution elicits release of the "readily releasable pool" (RRP) of vesicles through mechanisms that are independent of action potentials and calcium influx (Rosenmund and Stevens, 1996). Puff application of control extracellular solution supplemented with 500 mM sucrose evoked a barrage of synaptic events, giving rise to a postsynaptic response that could be blocked by the $\mathrm{GABA}_{\mathrm{A}} \mathrm{R}$ antagonist SR95531 (Fig. 3A). The sucrose-evoked response decayed back toward baseline during the puff, and immediately subsequent puffs elicited greatly diminished responses (Fig. 3B), suggesting that most of the RRP was depleted during a single application. The depletion gradually recovered such that consistent responses could be elicited at $1 \mathrm{~min}$ intervals (Fig. 3D). THA 

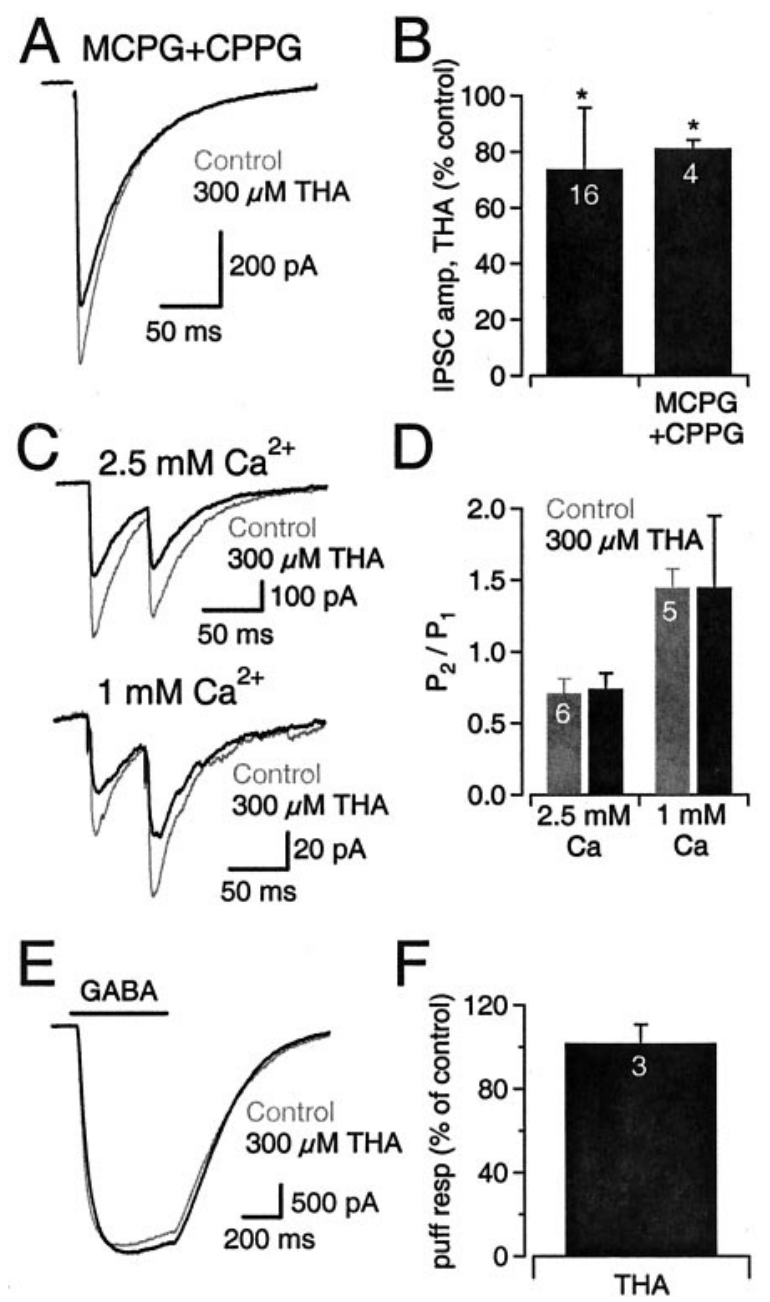

Figure 2. THA does not affect release probability or postsynaptic $G A B A_{A}$ receptors. $A$, In the presence of group I and II and group III metabotropic glutamate receptor antagonists (MCPG, $500 \mu \mathrm{m}$; and (PPG, $200 \mu \mathrm{m}$, respectively), THA still reduced evoked IPSCs. B, Bar graph comparing the effects of THA alone and in the presence of mGluR antagonists. amp, Amplitude. $C$, Effects of $300 \mu \mathrm{M}$ THA on IPSCs elicited by a pair of stimuli (50 msec interval). Paired pulse depression was observed in normal ( $2.5 \mathrm{~mm}$ ) calcium (top), and paired pulse facilitation was observed in low (1 mM) calcium (bottom). THA (black) reduced IPSC amplitudes compared with control ( gray) but did not affect the paired pulse ratio. D, Summary bar graph showing that THA (black bars) affected neither paired pulse depression nor facilitation. $E_{\text {, Currents elicited in a }}$ pyramidal cell by puff application of GABA in control solution (gray) and in the presence of 300 $\mu \mathrm{M}$ THA (black; THA was included in the bath and puffer solutions). F, Summary graph showing that THA did not affect $G A B A_{A}$ receptors on pyramidal cells. In all bar graphs, white numbers indicate the numbers of cells tested, and error bars indicate SD.

reduced the postsynaptic response to hyperosmotic stimulation (to $76 \pm 9 \%$ of control; $n=9 ; p<0.0001$; Fig. $3 C, D$ ), consistent with a reduction in the total amount of GABA packaged into the vesicles composing the RRP. The onset and extent of the effect of THA on electrically and sucrose-evoked IPSCs were similar (compare Figs. 1C, 3D), even though each sucrose application depleted the RRP, and electrical stimulation did not. These results, together with those showing that the effect of THA on IPSCs is not activity-dependent (Fig. 1C), are consistent with the idea that modulation by glutamate uptake of vesicular GABA content does not require release and recycling of synaptic vesicles.

Blocking glutamate uptake reduces miniature IPSC amplitudes

Although the results presented thus far are consistent with blockade of glutamate uptake reducing GABA synthesis and, conse-
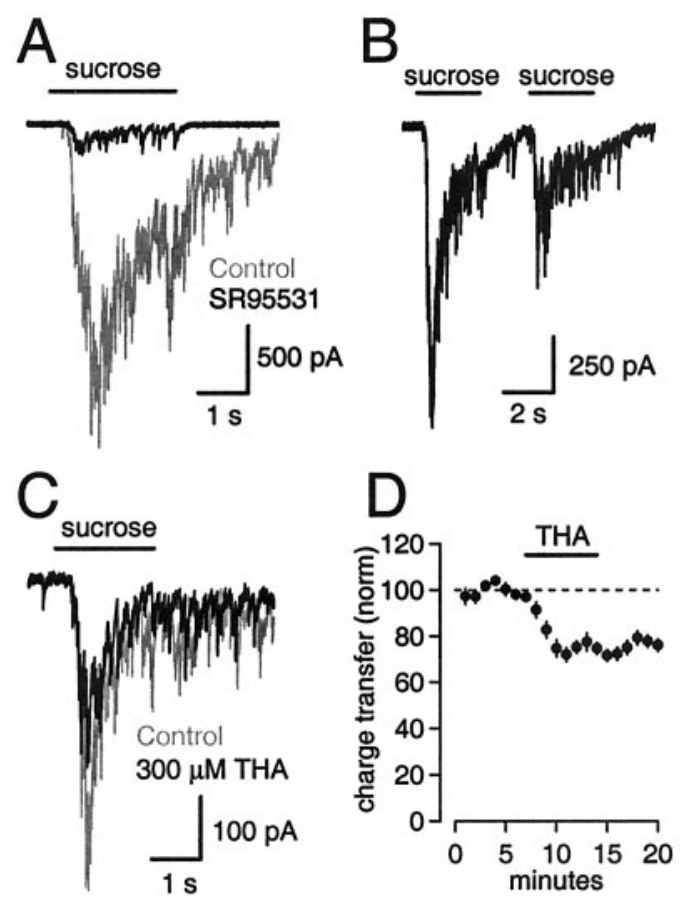

Figure 3. THA reduces hyperosmotically stimulated release of GABA. A, Postsynaptic current elicited by puff application of control solution with $500 \mathrm{~mm}$ sucrose (gray). The puffer was directed toward the pyramidal cell body. The response was almost completely abolished when the $\mathrm{GABA}_{A}$ antagonist SR95531 (10 $\mu \mathrm{m}$ ) was added to the bath (black). $B$, The responses to a second puff application of sucrose $1 \mathrm{sec}$ after the first indicated that the releasable pool only partially recovered from depletion by the first puff. C, Response to hyperosmotic stimulation in control solution (gray) and in the presence of $300 \mu \mathrm{M} \mathrm{THA} \mathrm{(black).D,} \mathrm{Normalized} \mathrm{charge} \mathrm{transfer}$ during sucrose-evoked IPSCs in the presence of $300 \mu \mathrm{M}$ THA $(n=9$ cells). THA was not included in the sucrose solution. Error bars indicate SEM.

quently, the amount of transmitter packaged into each vesicle, the effects of THA also could be explained by a reduction in the total number of synaptic vesicles available for release. In other systems, manipulations that increase or decrease vesicle filling cause commensurate changes in the amplitude of miniature synaptic events (Song et al., 1997; Pothos et al., 1998; Van der Kloot et al., 2000), suggesting that vesicles need not contain a prescribed amount of transmitter to be released. Moreover, mIPSCs recorded from hippocampal pyramidal cells in rats in which EAAC1 had been knocked down with chronic exposure to antisense oligonucleotides were smaller than those recorded in control animals (Sepkuty et al., 2002). Conversely, knocking out the synaptically localized GAD isoform GAD65 did not affect amplitude but decreased the frequency of $\mathrm{GABA}_{\mathrm{A}} \mathrm{R}$-mediated mIPSCs (Tian et al., 1999), possibly because only the "full" vesicles were released. To distinguish between these alternatives, we tested the effects of glutamate transporter blockade on postsynaptic responses to the release of single vesicles. Extracellular calcium was replaced with strontium to desynchronize electrically evoked release and thereby to resolve postsynaptic responses to individual release events occurring at stimulated synapses (Goda and Stevens, 1994) (Fig. 4A). In some cells, relatively high spontaneous release rates made it difficult to ensure that all events occurring within $700 \mathrm{msec}$ of stimulation arose at stimulated synapses (the first $100 \mathrm{msec}$ of the response, comprising primarily the synchronous IPSC, was excluded). However, the size and shape of presumptive evoked events in these cells, as well as the effects of various experimental manipulations described below, were indistinguishable from those obtained in cells with lower spontaneous 

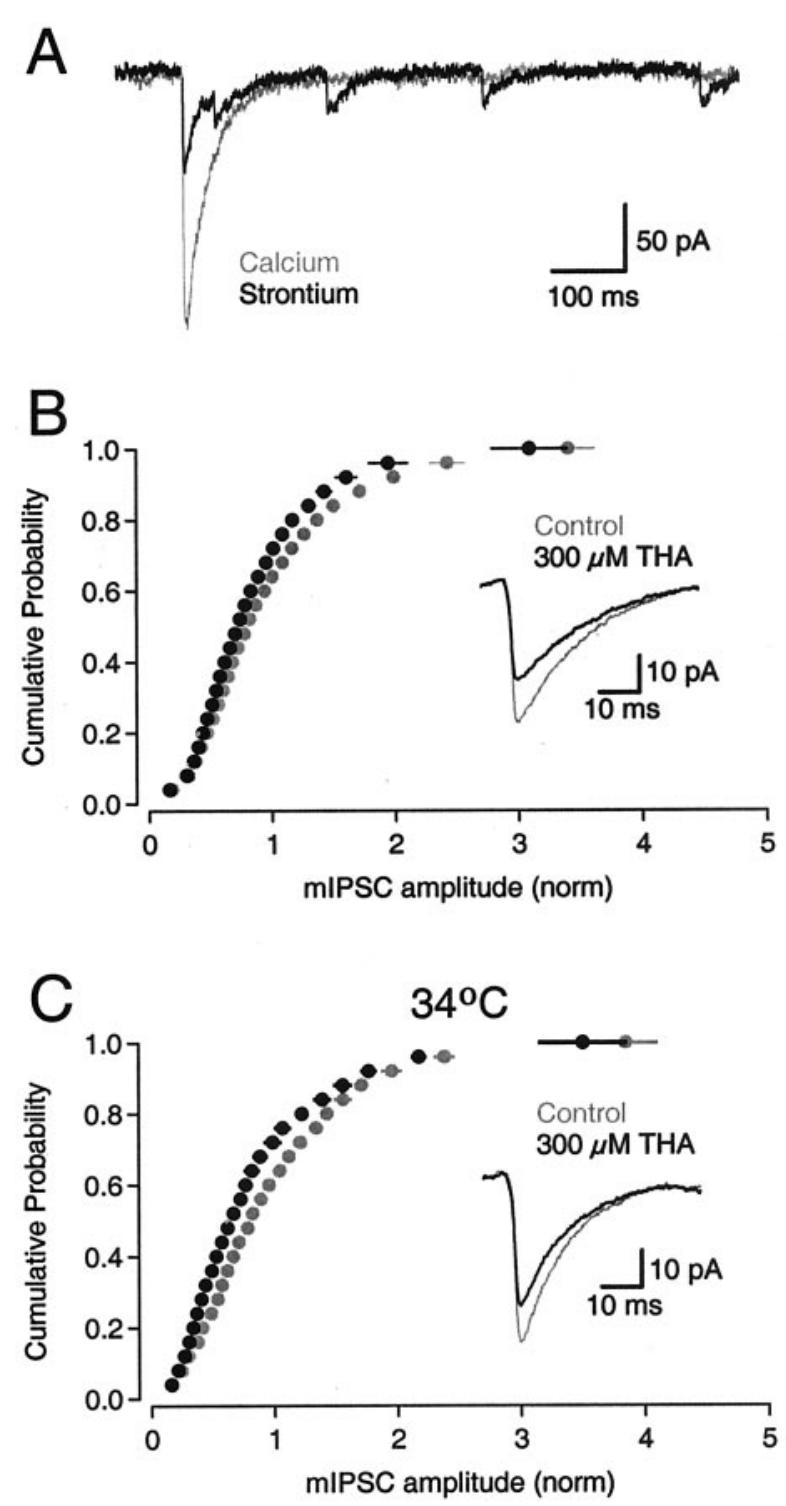

Figure 4. THA reduces mIPSC amplitudes. A, Electrically evoked IPSCs in normal calcium (gray) and in the same cell after calcium was replaced with strontium (black). In the presence of strontium, a diminished synchronous component of the response was followed by asynchronously released events, which were collected and pooled for each cell under each experimental condition. $B$, mIPSC amplitudes in control (strontium) solution (gray symbols; $n=1624$ events) and in the additional presence of $300 \mu \mathrm{M}$ THA (blacksymbols; $n=1491$ events). Events in each of 11 cells were normalized (norm) to the mean mIPSC amplitude in control, and cumulative probability histograms were placed into 25 equal bins ( $p$ between 0 and 1 ), allowing events in different cells to be pooled. Error bars indicate cell-to-cell variability (SEM) of the normalized histograms. Inset, Average mIPSC waveforms from a representative cell in control solution (gray) and in the presence of THA (black). C, mIPSC amplitudes in control (strontium) solution ( gray symbols; $n=909$ events) and in the additional presence of $300 \mu \mathrm{M}$ THA (black symbols; $n=958$ events) recorded at $34^{\circ} \mathrm{C}$. Inset, Average mIPS ( waveforms from a representative cell in control solution (gray) and in the presence of THA (black).

activity, so the results were pooled, and the events will be referred to as mIPSCs. THA $(300 \mu \mathrm{M})$ reduced the mean mIPSC amplitudes obtained in 11 cells from $32 \pm 5$ to $28 \pm 3 \mathrm{pA}$, or by $\sim 13 \%$ (Fig. $4 B$, inset). Individual mIPSCs normalized to the mean for each cell were then pooled, and cumulative probability histograms were generated, demonstrating a significant shift in the amplitude distribution ( $p<0.0001$, Mann-Whitney $U$ test; $n=$ 1491 in control and 1624 in THA; Fig. 4 B). Using identical selection criteria, similar numbers of events were obtained in each condition, suggesting that a change in MIPSC frequency did not occur in THA. The decay time constants of the average mIPSCs from individual cells were not altered by THA $(24.7 \pm 9.6 \mathrm{msec}$ in control vs $23.8 \pm 4.8 \mathrm{msec}$ in THA; $p=0.8 ; n=11$ cells), indicating that altered membrane properties did not account for the amplitude changes.

Neurotransmitter uptake and synthesis involve a series of temperature-dependent enzymatic reactions. The experiments presented thus far were performed at room temperature; at physiological temperatures, the increased capacity of other processes may reduce the impact of glutamate uptake on GABA synthesis, even though glutamate uptake itself is steeply temperaturedependent (Wadiche and Kavanaugh, 1998). At $34^{\circ} \mathrm{C}$, however, THA reduced mIPSC amplitudes to $84 \pm 8 \%$ of control in five cells (pooled data, $p<0.0001 ; n=909$ in control and 958 in THA; Fig. 4C), similar to the effects at room temperature, suggesting that glutamate uptake contributes to GABA synthesis at more physiological temperatures. Consistent with recent studies using molecular approaches to knock down neuronal glutamate transporters (Sepkuty et al., 2002), these results suggest that reducing glutamate uptake leads to a decrease in intraterminal glutamate levels and, as a result, a lower concentration of GABA in synaptic vesicles.

If filling of synaptic vesicles depends in part on newly synthesized GABA, and glutamate transporters provide the substrate for GABA synthesis, one would expect that incubation with MPA, which reduced IPSC amplitudes, would also reduce mIPSC amplitudes. The mean amplitude of pooled mIPSCs recorded in nine cells that had been preincubated with the GAD inhibitor MPA was $16 \%$ smaller than the mean from 19 pooled controls, and their distribution was significantly changed $(p<0.0001$, Mann-Whitney $U$ test; $n=3483$ for control and 1758 for MPA; data not shown).

\section{Exogenous glutamate application increases mIPSC amplitudes}

The data presented in Figure 4 indicate that reducing glutamate uptake into inhibitory terminals reduces GABA synthesis and, consequently, the amount of GABA released from synaptic vesicles. These results raise the possibility that increasing glutamate uptake may enhance GABAergic transmission, provided that postsynaptic $\mathrm{GABA}_{\mathrm{A}}$ Rs are not normally saturated during a synaptic event (Frerking et al., 1995; Nusser et al., 1997; Hajos et al., 2000; Perrais and Ropert, 2000). Such a mechanism could increase inhibition and thereby offer protection against hyperexcitability associated with pathological increases in extracellular glutamate levels. To test this idea, glutamate was added to the superfusion solution while mIPSCs were evoked in the presence of $\mathrm{Sr}^{2+}$ (Fig. 5). Addition of $100 \mu \mathrm{M}$ glutamate increased mean mIPSC amplitudes in 10 cells to $120 \pm 25 \%$ of control (pooled data, $p<0.0001$, Mann-Whitney $U$ test; $n=1990$ in control and 1975 in glutamate; Fig. 5A). A higher concentration $(500 \mu \mathrm{M})$ increased mIPSC amplitudes in nine cells even more (156 $\pm 41 \%$ of control; pooled data, $p<0.0001 ; n=1493$ in control and 1477 in glutamate; Fig. $5 A$ ). These increases appeared to reflect enhanced GABA synthesis, because $500 \mu \mathrm{M}$ glutamate exerted a much smaller effect in slices preincubated with $250 \mu \mathrm{M} \mathrm{MPA}$ (mean mIPSCs from 10 cells, $117 \pm 27 \%$ of control; Fig. $5 B$ ). Moreover, the effect of $100 \mu \mathrm{M}$ glutamate was abolished when it was applied together with THA $(300 \mu \mathrm{M})$ in five cells $(300 \mu \mathrm{M}$; $102 \pm 23 \%$ of control; pooled data, $p=0.95 ; n=1088$ in control and 1418 in glutamate plus THA; Fig. 5C). Applied alone, glutamate $(100 \mu \mathrm{M})$ also caused an amplitude increase in mIPSCs 

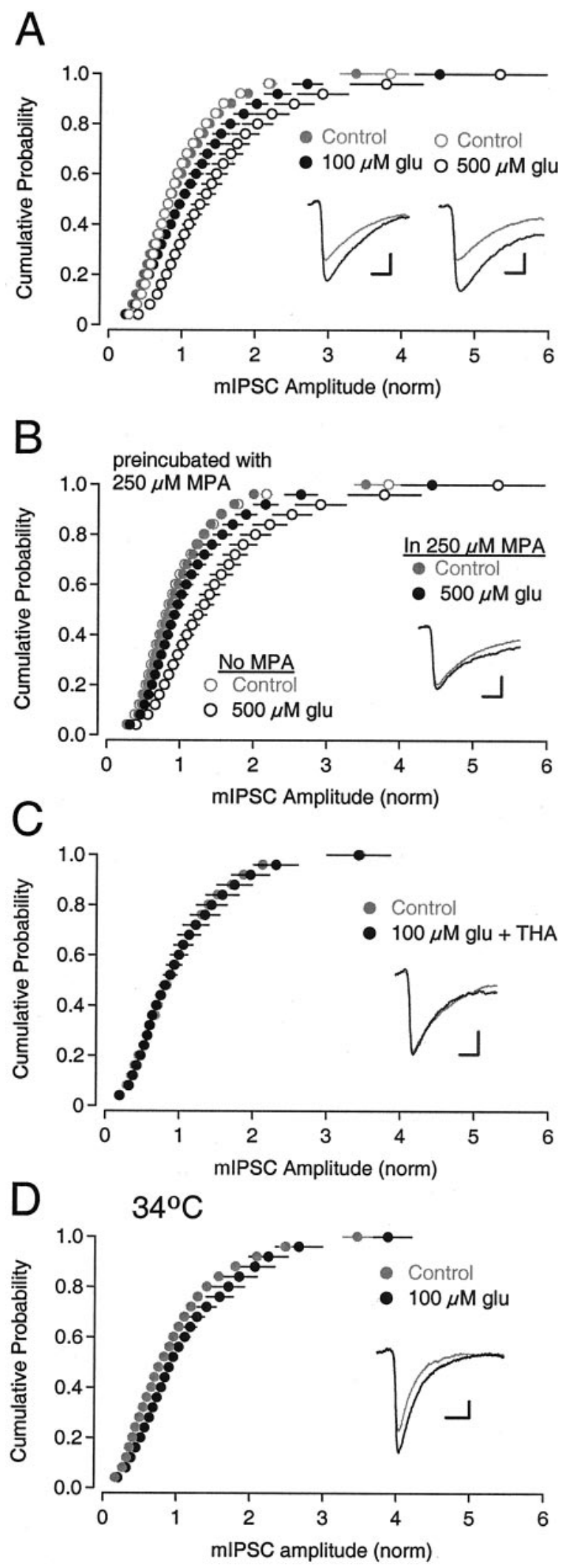

Figure 5. Exogenously applied glutamate increases mIPSC amplitudes. A, Addition of 100 $\mu \mathrm{M}$ (black, closed circles; $n=1975$ events) or $500 \mu \mathrm{M}$ (black, open circles; $n=1477$ events) glutamate $(g / u)$ increased mIPSC amplitude compared with control (gray, closed circles; $n=$ 1990; gray, open circles; $n=1493$, respectively). Cumulative probability histograms were constructed as described in the Figure $4 B$ legend. Inset, Average mIPSC waveforms from two representative cells in control solution (gray) and in the presence of either $100 \mu \mathrm{m}$ (black, left) or $500 \mu \mathrm{m}$ (black, right) glutamate. B, Preincubation with MPA, a GAD inhibitor, reduced the effect recorded at $34^{\circ} \mathrm{C}(116 \pm 22 \%$ of control in six cells; pooled data, $p<0.0001 ; n=973$ in control and 1177 in glutamate; Fig. 5D).

\section{Presynaptic uptake does not exhibit GLT-1 pharmacology}

A selective blocker of GLT-1, DHK (Arriza et al., 1994) inhibits glutamate uptake into excitatory synaptic terminals (Gundersen et al., 1993; Danbolt, 2001). Recently, a splice variant of GLT-1 that may be localized presynaptically on neurons was identified (Chen et al., 2002), suggesting a role in supplying excitatory terminals with glutamate. We used DHK to test whether the presynaptic transporter on inhibitory terminals is GLT-1-like. If it were, DHK would be expected to exert effects similar to those of THA. Alternatively, if DHK blocked only astrocytic transporters, it might be expected to raise extracellular glutamate (Muñoz et al., $1987)$ and to mimic exogenous glutamate.

The effects of DHK $(300 \mu \mathrm{M})$ on evoked IPSCs were complex (data not shown), possibly confounded by effects on release probability through activation of presynaptic kainate receptors (Kamboj et al., 1992; Rodriguez-Moreno et al., 1997). Because mIPSC amplitudes should not be affected by changes in release probability, we examined the effect of DHK on mIPSCs. DHK increased mIPSC amplitudes to $132 \pm 20 \%$ of control in eight cells (pooled data, $p<0.0001 ; n=1455$ in control and 1409 in DHK; Fig. 6A). The effect of DHK on mIPSC amplitudes was attributable to increased GABA synthesis, because no changes were observed in slices preincubated in $250 \mu \mathrm{M} \mathrm{MPA} \mathrm{(100} \pm 9 \%$ of control in five cells; pooled data, $p=0.6 ; n=790$ in control and 1096 in DHK; Fig. 6B). The striking difference between the effects of DHK and THA indicates that the presynaptic transporter on inhibitory terminals is not DHK-sensitive and therefore not a GLT-1 isoform. Furthermore, the effect of DHK is consistent with the hypothesis that elevating extracellular glutamate levels by blocking glial transport (Muñoz et al., 1987) enhances glutamate uptake into neurons and, consequently, increases GABA synthesis. These opposing effects of THA and DHK on mIPSC amplitudes (Figs. 4, 6) suggest that alterations of glutamate uptake into inhibitory presynaptic terminals may downregulate or upregulate the amount of GABA contained in synaptic vesicles and thereby regulate inhibitory synaptic strength.

\section{Discussion}

The findings presented here indicate that glutamate transporters regulate the synaptic pool of GABA in hippocampal CA1 interneurons. Reducing glutamate uptake diminished evoked IPSCs and mIPSCs without affecting postsynaptic receptors, suggesting that vesicular GABA content was reduced. These effects required GABA synthesis but not glial glutamate metabolism, suggesting that glutamate is taken up directly into inhibitory terminals and converted to GABA, which is then packaged into synaptic vesi-

\footnotetext{
of $500 \mu \mathrm{m}$ glutamate on mIPSC amplitude. The data for $500 \mu \mathrm{m}$ glutamate from $A$ (open circles) are included for comparison. Inset, Average mIPSC waveforms from a representative cell preincubated in MPA in control solution (gray) and in the presence of $500 \mu \mathrm{m}$ glutamate (black). C, Three hundred micromolar THA blocked the effect of glutamate on mIPSCs. mIPSCs recorded during coapplication of $300 \mu \mathrm{M}$ THA and $100 \mu \mathrm{m}$ glutamate (black symbols; $n=1418$ events) were not different compared with control (gray symbols; $n=1088$ events). Inset, Average $\mathrm{mIPSC}$ waveforms from a representative cell in control solution ( $g$ ray) and in the presence of 300 $\mu \mathrm{M}$ THA and $100 \mu \mathrm{m}$ glutamate (black). D, Recorded at $34^{\circ} \mathrm{C}$, addition of $100 \mu \mathrm{m}$ glutamate (black symbols; $n=1177$ events) increased mIPSC amplitude compared with control (gray symbols; $n=973$ events). Inset, Average mIPS ( waveforms from a representative cell in control solution (gray) and in the presence of $100 \mu \mathrm{m}$ glutamate (black). norm, Normalized.
} 

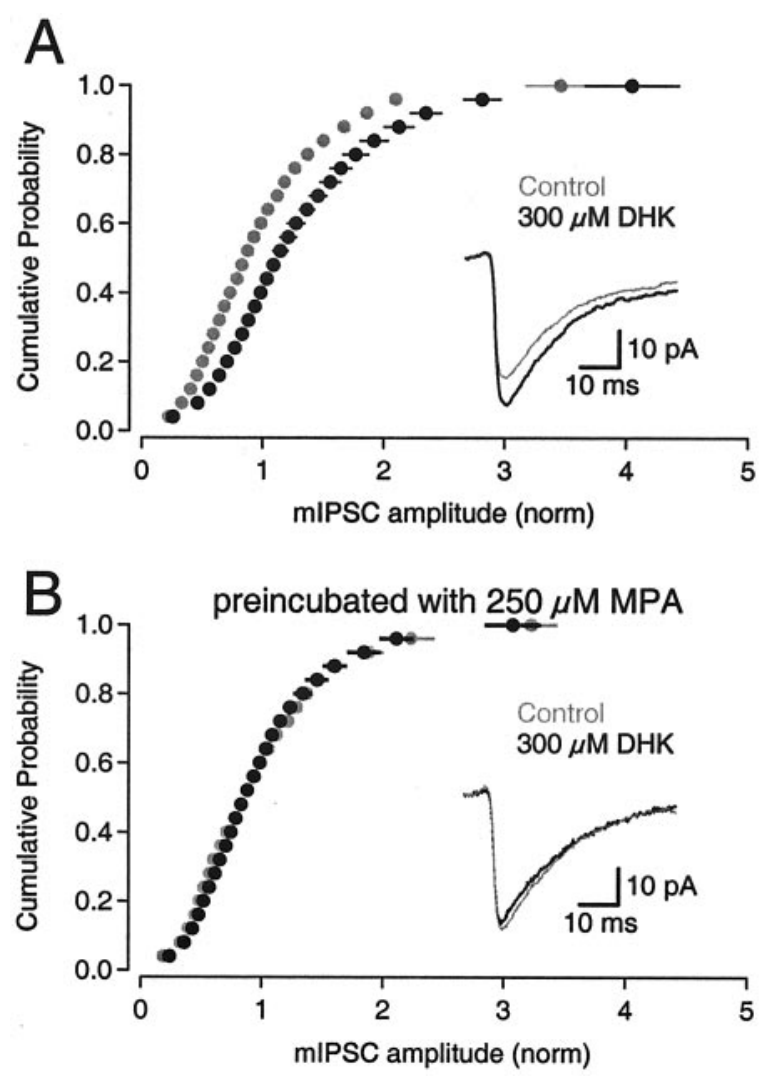

Figure 6. Presynaptic glutamate transporters do not exhibit GLT-1 pharmacology. A, Addition of $300 \mu \mathrm{m}$ DHK (black symbols; $n=1409$ events), a selective GLT-1 blocker, increased mIPSC amplitudes compared with control ( gray symbols; $n=1455$ events). Inset, Average mIPSC waveforms from a representative cell in control solution (gray) and in the presence of DHK (black). B, After preincubation with MPA, $300 \mu \mathrm{m}$ DHK (black; $n=1096$ events) no longer increased mIPSC amplitudes compared with control (gray; $n=790$ events). Inset, Average mIPSC waveforms from a representative cell in control solution (gray) and in the presence of DHK (black). norm, Normalized.

cles. Enhancement of mIPSCs by exogenous glutamate also required glutamate uptake and GABA synthesis. Previous work has shown that modulating GABA metabolism influences mIPSC amplitudes (Engel et al., 2001); our data point to glutamate uptake as an endogenous mechanism for bidirectional modulation of GABA synthesis and, consequently, inhibitory synaptic strength. GABA synthesis in vivo is decreased after knockdown of EAAC1 expression (Sepkuty et al., 2002), arguing that this modulation is not limited to the slice preparation. Manipulations of glutamate uptake exerted their effects on GABA release quickly and independently of synaptic activity, suggesting that presynaptic modulation of quantal size does not require vesicle release and recycling and that inhibition may be regulated rapidly. Reducing glutamate transport or GABA synthesis reduced mIPSC amplitudes only partially, however, indicating that other sources of GABA (e.g., reuptake) maintain the majority of the synaptic pool of transmitter. The primary role of glutamate transporters on inhibitory terminals, then, may be to strengthen inhibitory synapses in response to local increases in extracellular glutamate.

\section{Neuronal glutamate transporters provide a substrate for GABA synthesis}

Although astrocytic glutamate transporters (GLT-1 and GLAST) mediate the large majority of uptake in brain tissue (Rothstein et al., 1996; Tanaka et al., 1997; Diamond and Jahr, 2000), evidence also suggests that glutamate is taken up directly into nerve terminals (Gundersen et al., 1993; Danbolt, 2001). EAAC1 is expressed at inhibitory presynaptic terminals (Rothstein et al., 1994; He et al., 2000), where it could supply a substrate for GABA synthesis (Rothstein et al., 1994). In vivo studies demonstrate that infusion of EAAC1 antisense oligonucleotides into rat brain reduces both GABA content and de novo GABA synthesis in the hippocampus (Sepkuty et al., 2002). Because no selective inhibitor for EAAC1 exists, its physiological role in inhibitory transmission has been difficult to determine. THA and TBOA inhibit uptake by all of the transporter subtypes, whereas DHK (at the concentration used here) blocks only GLT-1. Together with the biochemical and anatomical studies mentioned above, our results showing that THA but not DHK reduces the synaptic GABA pool suggest that uptake into inhibitory terminals is mediated by EAAC1 or a transporter with similar pharmacology.

Glutamate taken up by astrocytes is converted by glutamine synthetase into glutamine, which is released and taken up into axon terminals, most likely by system A transporters (Chaudhry et al., 2002). In neurons, glutamate is produced from glutamine by phosphate-activated glutaminase (PAG). Some GABAergic neurons express PAG (Manns et al., 2001), whereas others do not (Kaneko and Mizuno, 1994), and it is unclear whether GABAergic neurons use this pathway to produce glutamate for GABA synthesis. Several of our findings argue against the possibility that THA indirectly reduced the supply of glutamate to inhibitory neurons by inhibiting astrocytic uptake. First, the glutamine synthetase inhibitor MSO, which disrupts astrocytic glutamate metabolism (Laake et al., 1995) and the supply of glutamine to presynaptic terminals (Rothstein and Tabakoff, 1984; Laake et al., 1995), did not diminish the effect of THA. Second, although in rats at this age, DHK and THA, at the concentrations used, exert comparable effects on glial transporter currents (Bergles and Jahr, 1997), DHK did not reduce mIPSC amplitudes (Fig. 6). The increase in mIPSC amplitudes in the presence of DHK argues that blocking astrocytic uptake led to higher glutamate concentrations near inhibitory terminals (Muñoz et al., 1987), thereby increasing neuronal uptake and GABA synthesis, in agreement with biochemical studies (Sepkuty et al., 2002). Although it is possible that glutamine may serve as a precursor for glutamate in inhibitory terminals, it does not appear to mediate the effects of glutamate transporter antagonists described here.

Inhibiting GAD with MPA reduced mIPSC amplitudes, but even prolonged incubation in MPA did not completely deplete GABA in synaptic terminals. The lack of detectable GABA levels in GAD 65/67 double knock-out mice (Ji et al., 1999) indicates that nearly all GABA is synthesized by GAD; the incomplete effects of MPA suggest that, once synthesized, GABA is recycled effectively for an extended period, presumably by GABA reuptake.

\section{Vesicular GABA content is dynamically regulated}

We have shown that the GABA content of vesicles is downregulated or upregulated by glutamate uptake in a way that is linked to GABA synthesis, and that incompletely filled vesicles, or "overfilled" vesicles, can be released. This is in agreement with studies showing that modulation of vesicular content can increase or decrease miniature postsynaptic event amplitudes (Song et al., 1997; Williams, 1997; Zhou et al., 2000). Enhancing glutamate uptake increased mIPSC amplitudes (Fig. 5), suggesting that CA1 synapses normally may not be saturated by synaptically released transmitter (Hajos et al., 2000). Our results showing that the releasable pool of synaptic vesicles did not have to be recycled 
before an effect of THA was observed (Fig. 1C) support the view that the vesicular transmitter concentration reflects an equilibrium between the vesicle lumen and the terminal cytoplasm (Williams, 1997). As a result, changes in GABA synthetic rate may be expressed very quickly in the vesicular transmitter concentration.

In light of these conclusions, it is surprising that we were unable to wash out the effects of THA on inhibitory synaptic responses, and, although glutamate-induced increases in mIPSC amplitude usually recovered, subsequent glutamate applications caused inconsistent results (data not shown). Modulating the extent of glutamate uptake may cause secondary metabolic effects that recover much more slowly. Such actions, however, may be specific to inhibitory terminals; the effects of transporter blockade on glutamatergic synaptic responses in astrocytes and pyramidal cells appear to wash out more readily (e.g., Bergles and Jahr, 1997; Diamond, 2001).

\section{A new protective role for glutamate transporters}

The broad clinical utility of benzodiazapines, which exert only modest effects on GABAergic transmission (Perrais and Ropert, 2000), indicates that even small changes in inhibitory strength have a significant physiological impact. Data reported here and elsewhere (Sepkuty et al., 2002) suggest that suppression of neuronal transporters, perhaps by modulatory proteins (Lin et al., 2001), could reduce inhibition. Our results showing that exogenous glutamate enhances mIPSCs by way of neuronal transporters (Fig. 5) argue that glutamate uptake into inhibitory terminals may function as a mechanism to upregulate inhibition under certain conditions. For example, transient increases in extracellular glutamate levels that occur during hypoxia or seizures (for review, see Danbolt, 2001) may result in a compensatory or protective increase in inhibitory synaptic strength.

It also seems probable that such modulation occurs even in nonpathological conditions: Knockdown of EAAC1 expression in rats with antisense DNA induces epilepsy (Rothstein et al., 1996; Sepkuty et al., 2002). This may indicate that the loss of the protective mechanism proposed here may itself be epileptogenic, although EAAC1 also may limit excitability in other ways (Diamond, 2001). Evidence from human studies indicates that extracellular glutamate rises in the hippocampus before seizure onset (During and Spencer, 1993). If this were generally true in epilepsy, then glutamate transporters could play an important role in preventing seizures. In addition to changes in extracellular glutamate, changes in the surface expression and capacity of transporters on terminals could play a role in regulating inhibitory synapses.

\section{References}

Arnth-Jensen N, Jaboudon D, Scanziani M (2002) Cooperation between independent hippocampal synapses is controlled by glutamate uptake. Nat Neurosci 5:325-331.

Arriza JL, Fairman WA, Wadiche JI, Murdoch GH, Kavanaugh MP, Amara SG (1994) Functional comparisons of three glutamate transporter subtypes cloned from human motor cortex. J Neurosci 14:5559-5569.

Asztely F, Erdemli G, Kullmann DM (1997) Extrasynaptic glutamate spillover in the hippocampus: dependence on temperature and the role of active glutamate uptake. Neuron 18:281-293.

Bergles DE, Jahr CE (1997) Synaptic activation of glutamate transporters in hippocampal astrocytes. Neuron 19:1297-1308.

Brasnjo G, Otis TS (2001) Neuronal glutamate transporters control activation of postsynaptic metabotropic glutamate receptors and influence cerebellar long-term depression. Neuron 31:607-616.

Carter AG, Regehr WG (2000) Prolonged synaptic currents and glutamate spillover at the parallel fiber to stellate cell synapse. J Neurosci 20:4423-4434.
Chaudhry FA, Schmitz D, Reimer RJ, Larsson P, Gray AT, Nicoll R, Kavanaugh M, Edwards RH (2002) Glutamine uptake by neurons: interaction of protons with system a transporters. J Neurosci 22:62-72.

Chen W, Aoki C, Mahadomrongkul V, Gruber CE, Wang GJ, Blitzblau R, Irwin N, Rosenberg PA (2002) Expression of a variant form of the glutamate transporter GLT1 in neuronal cultures and in neurons and astrocytes in the rat brain. J Neurosci 22:2142-2152.

Conti F, DeBiasi S, Minelli A, Rothstein JD, Melone M (1998) EAAC1, a high-affinity glutamate transporter, is localized to astrocytes and gabaergic neurons besides pyramidal cells in the rat cerebral cortex. Cereb Cortex 8:108-116.

Danbolt NC (2001) Glutamate uptake. Prog Neurobiol 65:1-105.

Desai MA, McBain CJ, Kauer JA, Conn PJ (1994) Metabotropic glutamate receptor-induced disinhibition is mediated by reduced transmission at excitatory synapses onto interneurons and inhibitory synapses onto pyramidal cells. Neurosci Lett 181:78-82.

Diamond JS (2001) Neuronal glutamate transporters limit activation of NMDA receptors by neurotransmitter spillover on CA1 pyramidal cells. J Neurosci 21:8328-8338.

Diamond JS, Jahr CE (2000) Synaptically released glutamate does not overwhelm transporters on hippocampal astrocytes during high-frequency stimulation. J Neurophysiol 83:2835-2843.

During M, Spencer D (1993) Extracellular hippocampal glutamate and spontaneous seizure in the conscious human brain. Lancet 341:1607-1610.

Engel D, Schmitz D, Gloveli T, Frahm C, Heinemann U, Draguhn A (1998) Laminar difference in GABA uptake and GAT-1 expression in rat CA1. J Physiol (Lond) 512:643-649.

Engel D, Pahner I, Schulze K, Frahm C, Jarry H, Ahnert-Hilger G, Draguhn A (2001) Plasticity of rat central inhibitory synapses through GABA metabolism. J Physiol (Lond) 535:473-482.

Fitzsimonds RM, Dichter MA (1996) Heterologous modulation of inhibitory synaptic transmission by metabotropic glutamate receptors in cultured hippocampal neurons. J Neurophysiol 75:885-893.

Frerking M, Borges S, Wilson M (1995) Variation in GABA mini amplitude is the consequence of variation in transmitter concentration. Neuron 15:885-895.

Gereau RW, Conn PJ (1995) Multiple presynaptic metabotropic glutamate receptors modulate excitatory and inhibitory synaptic transmission in hippocampal area CA1. J Neurosci 15:6879-6889.

Goda Y, Stevens CF (1994) Two components of transmitter release at a central synapse. Proc Natl Acad Sci USA 91:12942-12946.

Gundersen V, Danbolt NC, Ottersen OP, Storm-Mathisen J (1993) Demonstration of glutamate/aspartate uptake activity in nerve endings by use of antibodies recognizing exogenous D-aspartate. Neuroscience 57:97-111.

Hajos N, Nusser Z, Rancz EA, Freund TF, Mody I (2000) Cell type- and synapse-specific variability in synaptic GABAA receptor occupancy. Eur J Neurosci 12:810-818.

He Y, Janssen WG, Rothstein JD, Morrison JH (2000) Differential synaptic localization of the glutamate transporter EAAC1 and glutamate receptor subunit GluR2 in the rat hippocampus. J Comp Neurol 418:255-269.

Hertz L, Dringen R, Schousboe A, Robinson SR (1999) Astrocytes: glutamate producers for neurons. J Neurosci Res 57:417-428.

Ji F, Kanbara N, Obata K (1999) GABA and histogenesis in fetal and neonatal mouse brain lacking both the isoforms of glutamic acid decarboxylase. Neurosci Res 33:187-194.

Kamboj R, Schoepp D, Nutt S, Shekter L, Korczak B, True R, Zimmerman D, Wosnick M (1992) Molecular structure and pharmacological characterization of humEAA2, a novel kainate receptor subunit. Mol Pharm 42:10-15.

Kaneko T, Mizuno N (1994) Glutamate-synthesizing enzymes in GABAergic neurons of the neocortex: a double immunofluorescence study in the rat. Neuroscience 61:839-849.

Laake JH, Slyngstad TA, Haug FM, Ottersen OP (1995) Glutamine from glial cells is essential for the maintenance of the nerve terminal pool of glutamate: immunogold evidence from hippocampal slice cultures. J Neurochem 65:871-881.

Lamar Jr C (1970) Mercaptopropionic acid: a convulsant that inhibits glutamate decarboxylase. J Neurochem 17:165-170.

Lin CI, Orlov I, Ruggiero AM, Dykes-Hoberg M, Lee A, Jackson M, Rothstein JD (2001) Modulation of the neuronal glutamate transporter EAAC1 by the interacting protein GTRAP3-18. Nature 410:84-88.

Lozovaya NA, Kopanitsa MV, Boychuk YA, Krishtal OA (1999) Enhance- 
ment of glutamate release uncovers spillover-mediated transmission by $\mathrm{N}$-methyl-D-aspartate receptors in the rat hippocampus. Neuroscience 91:1321-1330.

Manns ID, Mainville L, Jones BE (2001) Evidence for glutamate, in addition to acetylcholine and GABA, neurotransmitter synthesis in basal forebrain neurons projecting to the entorhinal cortex. Neuroscience 107:249-263.

Martin DL, Tobin AJ (2000) Mechanisms controlling GABA synthesis and degradation in the brain. In: GABA in the nervous system (Martin DL, Olsen RW, eds), pp 25-41. Philadelphia: Lippincott Williams \& Wilkins.

Martinez-Hernandez A, Bell KP, Norenberg MD (1977) Glutamine synthetase: glial localization in brain. Science 195:1356-1358.

McBain CJ, Fisahn A (2001) Interneurons unbound. Nat Rev Neurosci 2:11-23.

Mitchell SJ, Silver RA (2000) Glutamate spillover suppresses inhibition by activating presynaptic mGluRs. Nature 404:498-502.

Muñoz MD, Herreras O, Herranz AS, Solis JM, Martin del Rio R, Lerma J (1987) Effects of dihydrokainic acid on extracellular amino acids and neuronal excitability in the in vivo rat hippocampus. Neuropharmacology $26: 1-8$.

Nusser Z, Cull-Candy S, Farrant M (1997) Differences in synaptic GABA(A) receptor number underlie variation in GABA mini amplitude. Neuron 19:697-709.

Perrais D, Ropert N (2000) Altering the concentration of GABA in the synaptic cleft potentiates miniature IPSCs in rat occipital cortex. Eur J Neurosci 12:400-404.

Pothos EN, Davila V, Sulzer D (1998) Presynaptic recording of quanta from midbrain dopamine neurons and modulation of the quantal size. J Neurosci 18:4106-4118.

Reichelt W, Knopfel T (2002) Glutamate uptake controls expression of a slow postsynaptic current mediated by mGluRs in cerebellar Purkinje cells. J Neurophysiol 87:1974-1980.

Rodriguez-Moreno A, Herreras O, Lerma J (1997) Kainate receptors presynaptically downregulate GABAergic inhibition in the rat hippocampus. Neuron 19:893-901.

Rosenmund C, Stevens CF (1996) Definition of the readily releasable pool of vesicles at hippocampal synapses. Neuron 16:1197-1207.

Rothstein J, Tabakoff B (1984) Alteration of striatal glutamate release after glutamine synthetase inhibition. J Neurochem 43:1438-1446.

Rothstein JD, Martin L, Levey AI, Dykes-Hoberg M, Jin L, Wu D, Nash N,
Kuncl RW (1994) Localization of neuronal and glial glutamate transporters. Neuron 13:713-725.

Rothstein JD, Dykes-Hoberg M, Pardo CA, Bristol LA, Jin L, Kuncl RW, Kanai Y, Hediger MA, Wang Y, Schielke JP, Welty DF (1996) Knockout of glutamate transporters reveals a major role for astroglial transport in excitotoxicity and clearance of glutamate. Neuron 16:675-686.

Sakmann B, Stuart G (1995) Patch-pipette recordings from the soma, dendrites, and axon of neurons in brain slices. In: Single-channel recording, Ed 2 (Sakmann B, Neher E, eds), pp 199-211. New York: Plenum.

Sellinger OZ (1967) Inactivation of cerebral glutamine synthetase by DLmethionine-DL-sulfoximine. Biochim Biophys Acta 132:514-516.

Sepkuty JP, Cohen AS, Eccles C, Rafiq A, Behar K, Ganel R, Coulter DA, Rothstein JD (2002) A neuronal glutamate transporter contributes to neurotransmitter GABA synthesis and epilepsy. J Neurosci 22:6372-6379.

Shimamoto K, Lebrun B, Yasuda-Kamatani Y, Sakaitani M, Shigeri Y, Yumoto N, Nakajima T (1998) DL-Threo-beta-benzyloxyaspartate, a potent blocker of excitatory amino acid transporters. Mol Pharmacol 53:195-201.

Song H, Ming G, Fon E, Bellocchio E, Edwards RH, Poo M (1997) Expression of a putative vesicular acetylcholine transporter facilitates quantal transmitter packaging. Neuron 18:815-826.

Tanaka K, Watase K, Manabe T, Yamada K, Watanabe M, Takahashi K, Iwama H, Nishikawa T, Ichihara N, Kikuchi T, Okuyama S, Kawashima N, Hori S, Takimoto M, Wada K (1997) Epilepsy and exacerbation of brain injury in mice lacking the glutamate transporter GLT-1. Science 276:1699-1702.

Tian N, Petersen C, Kash S, Baekkeskov S, Copenhagen D, Nicoll R (1999) The role of the synthetic enzyme GAD65 in the control of neuronal gammaaminobutyric acid release. Proc Natl Acad Sci USA 96:12911-12916.

Van der Kloot W, Colasante C, Cameron R, Molgo J (2000) Recycling and refilling of transmitter quanta at the frog neuromuscular junction. J Physiol (Lond) 523:247-258.

Wadiche JI, Kavanaugh MP (1998) Macroscopic and microscopic properties of a cloned glutamate transporter/chloride channel. J Neurosci 18:7650-7661.

Williams J (1997) How does a vesicle know it is full? Neuron 18:683-686.

Zhou Q, Petersen CC, Nicoll RA (2000) Effects of reduced vesicular filling on synaptic transmission in rat hippocampal neurones. J Physiol 525: 195-206. 